\title{
Hospital outbreak of Pseudomonas aeruginosa producing extended-spectrum oxacillinase OXA-19
}

Pseudomonas aeruginosa is one of the leading causes of bacteraemia and pneumonia in patients hospitalized in intensive care units (Bertrand et al., 2001). In addition to being intrinsically resistant to several antimicrobial agents, $P$. aeruginosa can acquire resistance to conventional anti-pseudomonal antibiotics including anti-pseudomonal penicillins, ceftazidime, carbapenems, aminoglycosides and ciprofloxacin (Carmeli et al., 1999). The mechanisms of resistance to $\beta$-lactams mostly involve stable upregulation of the intrinsic cephalosporinase AmpC, acquisition of transferable $\beta$-lactamases (with narrow or extended spectrum), outer-membrane impermeability (alteration or underproduction of porin OprD) or MexAB-OprF efflux pump overproduction (Livermore, 2002). Clinical $P$. aeruginosa isolates producing class A extended-spectrum $\beta$-lactamases (ESBLs) such as TEM, SHV, PER, GES, VEB, BEL and CTX-M are frequently described (Weldhagen et al., 2003). Ambler class B enzymes (metallo- $\beta$-lactamases, such as IMP, VIM, SPM and GIM) also spread among clinical $P$. aeruginosa isolates, especially in Asia and Europe (Rossolini et al., 2007). P. aeruginosa strains producing extended-spectrum oxacillinases (from the Ambler class D) have sporadically been described but knowledge of their spread in the clinical setting remains poor (Poirel et al., 2010).

Within the framework of a hospital clinical research project, we found evidence of an outbreak of $P$. aeruginosa producing the extended-spectrum oxacillinase OXA-19. This report describes the microbiological investigation of this outbreak.

In the bacteriology laboratory of Nancy University Hospital (Lorraine, France), the susceptibility of all $P$. aeruginosa isolates to

The GenBank/EMBL/DDBJ accession number for the sequence of the class 1 integron is FJ906752. a panel of anti-pseudomonal agents was systematically determined by the disc diffusion method on Mueller-Hinton agar (bioMérieux) according to the guidelines of the Antibiogram Committee of the French Microbiology Society. All ceftazidime-resistant $\left(\mathrm{Caz}^{\mathrm{R}}\right)(\mathrm{MIC}>8 \mathrm{mg}$ $\left.1^{-1}\right) P$. aeruginosa strains, excluding cystic fibrosis isolates, were collected over 1 year (from October 2007 to September 2008). Overall, $92 \mathrm{Caz}^{\mathrm{R}}$ P. aeruginosa isolates were obtained from 48 patients.

An ESBL phenotypic screening test was performed for all $\mathrm{Caz}^{\mathrm{R}}$ isolates by using the double disc susceptibility test (DDST), where discs of ceftazidime, aztreonam and cefepime (30 $\mu \mathrm{g}$ each) were positioned at a variable distance from a disc containing amoxicillin $(20 \mu \mathrm{g})$ and clavulanate (10 $\mu \mathrm{g})$ (Jiang et al., 2006). A slight synergy was observed for 48 isolates between ceftazidime- and clavulanatecontaining discs only when the distance between discs (centre to centre) was $12 \mathrm{~mm}$ (data not shown).

For all of these isolates, isoelectric focusing (Hocquet et al., 2003) revealed the presence of the AmpC cephalosporinase (pI 8.1), as well as a secondary $\beta$-lactamase exhibiting a $\mathrm{pI}$ of 7.6. The identification of the secondary $\beta$-lactamase was carried out by PCR on whole-cell DNA using primers specific for the class $\mathrm{D} \beta$-lactamases (OXA10, OXA-2 and OXA-1 derivatives) because of the basic $\mathrm{pI}$ of this enzyme and the poor synergy provided by clavulanate toward ceftazidime (Bert et al., 2002). Only PCR using primers targeting the $b l a_{\mathrm{OXA}-10}$ group was positive. As $b l a_{\mathrm{OXA}}$ genes are most frequently found in class 1 integrons (Fluit \& Schmitz, 2004), we also performed PCR experiments using consensus primers $5^{\prime}$-CS and $3^{\prime}$-CS specific for class 1 integrons (Levesque et al., 1995). The PCR amplicons (1673 bp) were sequenced on both strands, resulting in the identification of a class 1 integron (GenBank accession no. FJ906752) containing two resistance gene cassettes, namely aacA4, which determines an aminoglycoside $6^{\prime}-N$ acetyltransferase conferring high resistance to gentamicin and tobramycin, and bla $a_{\text {OXA-19, }}$, which encodes the extendedspectrum OXA-19 (Mugnier et al., 1998).

PFGE typing was performed on all $\mathrm{Caz}^{\mathrm{R}}$ isolates as previously described (Bertrand et al., 2000). We used the GelCompar software (Applied Maths) to establish a DNA similarity matrix. A dendrogram was constructed using the unweighted pairgroup method with arithmetic averages clustering method and the Dice coefficient. We ensured that the gels were comparable by including Staphylococcus aureus NCTC 8325 as a reference. PFGE results were interpreted according to international recommendations (Tenover et al., 1995). PFGE analysis showed that the 92 isolates clustered in 10 different PFGE patterns: two large clusters comprising 23 and 14 isolates, respectively; three smaller clusters comprising three, two and three isolates, respectively; and four unique patterns from four isolates (Fig. 1). A single PFGE pattern was detected in 42 patients, and at least two different PFGE patterns were identified in six patients. The 48 isolates producing OXA-19 recovered from 27 patients were clustered into three PFGE patterns: no. 2 (three patients), no. 6 (one patient) and no. 8 (23 patients). One intensive care unit was the epicentre of the main OXA-19-producing clone (no. 8), which further spread to other units, likely as a consequence of patient transfers. Moreover, considering the epidemic curve, it is likely that the $b l a_{\mathrm{OXA}-19}$ gene was transferred from PFGE pattern 8 to PFGE patterns 2 and 6 (Fig. 2). Among the 27 non-replicate OXA-19-producing isolates (one per patient), 22 were resistant to all anti-pseudomonal antibiotics tested (ticarcillin, piperacillin-tazobactam, ceftazidime, cefepime, aztreonam, imipenem, meropenem, tobramycin, amikacin and ciprofloxacin) with the exception of colistin. 


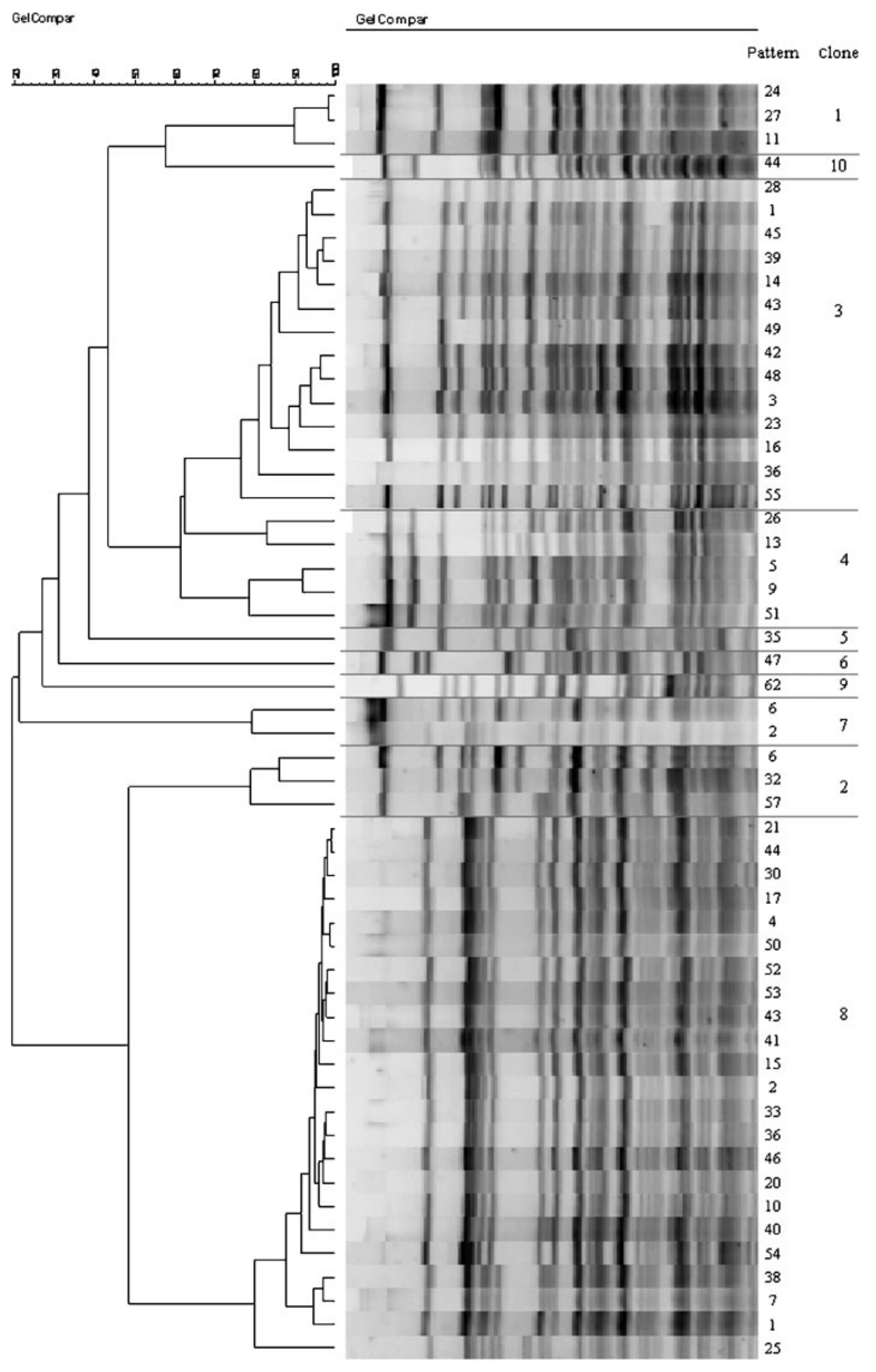

Fig. 1. Pulsed-field electrophoresis banding patterns after Dral digestion of non-duplicate $P$. aeruginosa isolates.

To our knowledge, this is the first report describing an outbreak involving $P$. aeruginosa isolates producing a clavulanate-resistant extended-spectrum oxacillinase. During the same period, we also evidenced an outbreak of $P$. aeruginosa producing OXA-28 in another French hospital (Hocquet et al., 2008). The emergence of $P$. aeruginosa producing such $\beta$-lactamases may be related to a recent spread of extended-spectrum oxacillinases among $P$. aeruginosa clinical isolates. This phenomenon may be underestimated because of the difficulty in detecting such strains in clinical laboratories. Indeed, most of these oxacillinases (with the exception of OXA-18) are poorly inhibited by clavulanate. This undermines DDST 


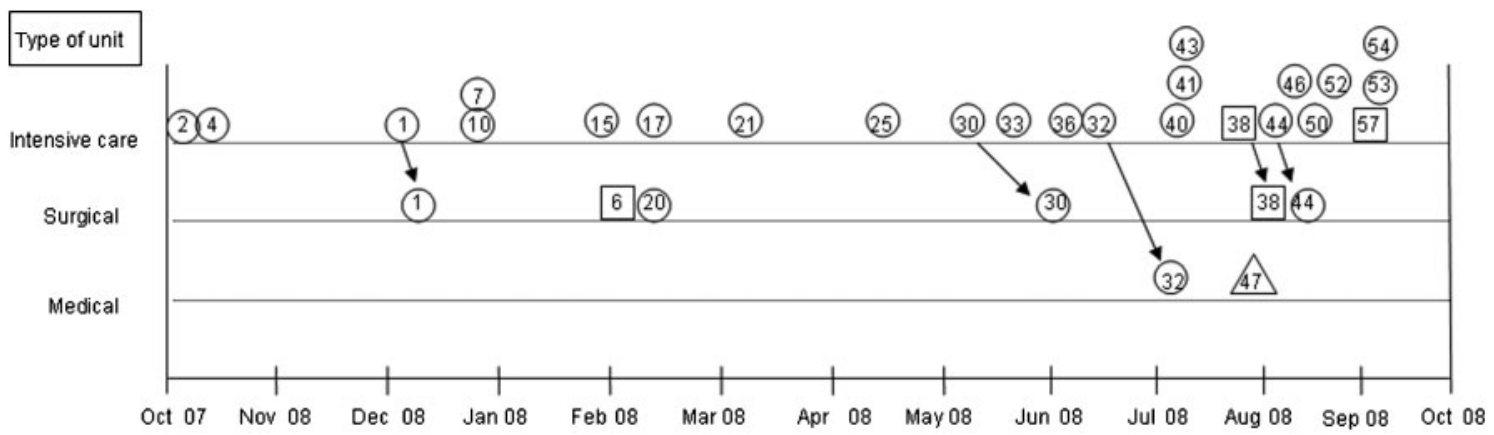

Fig. 2. Spatio-temporal distribution of the PFGE pattern of OXA-19-producing isolates. Numbers indicate patient number according to the date of first isolation; circles represent PFGE pattern 8; squares represent PFGE pattern 2; and the triangle represents PFGE pattern 6. Arrows indicate the transfer of the patient between units.

screening tests using conventional distance $(\geqslant 20 \mathrm{~mm})$ between third-generation cephalosporins and clavulanate. Extendedspectrum oxacillinase activity is also inhibited by cloxacillin, a compound used in phenotypic screening tests to unmask ESBLs in an AmpC overproduction background (Aubert et al., 2004). OXA-19producing $P$. aeruginosa is detected only when ceftazidime- and clavulanatecontaining discs are in close approximation to each other.

In conclusion, the emergence and spread of extended-spectrum oxacillinases among $P$. aeruginosa clinical strains is a matter of concern. These multidrug-resistant bacteria caused infections that are difficult to treat, as colistin often remains the unique therapeutic option. Ongoing surveillance of these micro-organisms, with the use of a specific screening test, is warranted as well as the implementation of infection control measures to prevent their cross-transmission between patients.

\section{Acknowledgements}

This work was funded by grants from the French Ministry of Health in the framework of a clinical research programme 2007.

\section{Pascal Cholley, ${ }^{1,2}$ Didier Hocquet, ${ }^{3,4}$ Corentine Alauzet, ${ }^{5}$ Aurélie Cravoisy-Popovic, ${ }^{6}$ Daniel Talon, ${ }^{1,2,4}$ Nejla Aissa, ${ }^{5}$ Patrick Plésiat ${ }^{3,4}$ and Xavier Bertrand ${ }^{1,2,4}$}

\footnotetext{
${ }^{1}$ Service d'Hygiène Hospitalière, Centre Hospitalier Universitaire Besançon, Besançon, France
}

${ }^{2}$ UMR 6249 Chrono-Environnement, Université de Franche-Comté, Besançon, France

${ }^{3}$ Service de Bactériologie, Centre Hospitalier Universitaire Besançon, Besançon, France

${ }^{4}$ Centre National de RéférenceRésistance chez $P$. aeruginosa, Centre Hospitalier Universitaire Besançon, Besançon, France

${ }^{5}$ Service de Bactériologie, Centre Hospitalier Universitaire Nancy, Nancy, France

'Service de Réanimation Médicale, Centre Hospitalier Universitaire Nancy, Nancy, France

Correspondence: Xavier Bertrand (xavier.bertrand@univ-fcomte.fr)

Aubert, D., Girlich, D., Naas, T., Nagarajan, S. \& Nordmann, P. (2004). Functional and structural characterization of the genetic environment of an extended-spectrum $\beta$-lactamase $b a_{\mathrm{VEB}}$ gene from a Pseudomonas aeruginosa isolate obtained in India. Antimicrob Agents Chemother 48, 32843290.

Bert, F., Branger, C. \& Lambert-Zechovsky, N. (2002). Identification of PSE and OXA $\beta$ lactamase genes in Pseudomonas aeruginosa using PCR-restriction fragment length polymorphism. $J$ Antimicrob Chemother 50, 11-18.

Bertrand, X., Bailly, P., Blasco, G., Balvay, P., Boillot, A. \& Talon, D. (2000). Large outbreak in a surgical intensive care unit of colonization or infection with Pseudomonas aeruginosa that overexpressed an active efflux pump. Clin Infect Dis 31, E9-E14.

Bertrand, X., Thouverez, M., Talon, D., Boillot, A., Capellier, G., Floriot, C. \& Helias, J. P. (2001). Endemicity, molecular diversity and colonisation routes of Pseudomonas aeruginosa in intensive care units. Intensive Care Med 27, 1263-1268.

Carmeli, Y., Troillet, N., Karchmer, A. W. \& Samore, M. H. (1999). Health and economic outcomes of antibiotic resistance in Pseudomonas aeruginosa. Arch Intern Med 159, 1127-1132.

Fluit, A. C. \& Schmitz, F. J. (2004). Resistance integrons and super-integrons. Clin Microbiol Infect 10, 272-288.

Hocquet, D., Bertrand, X., Kohler, T., Talon, D. \& Plesiat, P. (2003). Genetic and phenotypic variations of a resistant Pseudomonas aeruginosa epidemic clone. Antimicrob Agents Chemother 47, 1887-1894.

Hocquet, D., Thouverez, M., Legrand, F., Bertrand, X. \& Plesiat, P. (2008). Modulation of extended-spectrum beta-lactamase expression in response to antibiotic chemotherapy. In Abstracts of the 48th Annual Interscience Conference on Antimicrobial Agents and Chemotherapy/46th Annual Meeting of the Infectious Diseases Society of America, Abstract C1-118. Washington DC: American Society for Microbiology, Infectious Diseases Society of America.

Jiang, X., Zhang, Z., Li, M., Zhou, D., Ruan, F. \& Lu, Y. (2006). Detection of extended-spectrum $\beta$-lactamases in clinical isolates of Pseudomonas aeruginosa. Antimicrob Agents Chemother 50, 2990-2995.

Levesque, C., Piche, L., Larose, C. \& Roy, P. H. (1995). PCR mapping of integrons reveals several novel combinations of resistance genes. Antimicrob Agents Chemother 39, 185-191.

Livermore, D. M. (2002). Multiple mechanisms of antimicrobial resistance in Pseudomonas aeruginosa: our worst nightmare? Clin Infect Dis 34, 634-640.

Mugnier, P., Casin, l., Bouthors, A. T. \& Collatz, E. (1998). Novel OXA-10-derived extended-spectrum $\beta$-lactamases selected in vivo or in vitro. Antimicrob Agents Chemother 42, 3113-3116. 
Poirel, L., Naas, T. \& Nordmann, P. (2010).

Diversity, epidemiology and genetics of class D

$\beta$-lactamases. Antimicrob Agents Chemother 54,

24-38.

Rossolini, G. M., Mantengoli, E., Docquier, J. D., Musmanno, R. A. \& Coratza, G. (2007).

Epidemiology of infections caused by multiresistant gram-negatives: ESBLs, MBLs, panresistant strains. New Microbiol 30, 332-339.

Tenover, F. C., Arbeit, R. D., Goering, R. V., Mickelsen, P. A., Murray, B. E., Persing, D. H. \&

Swaminathan, B. (1995). Interpreting chromosomal DNA restriction patterns produced by pulsed-field gel electrophoresis: criteria for bacterial strain typing. J Clin Microbiol 33, 2233-2239.

Weldhagen, G. F., Poirel, L. \& Nordmann, P. (2003). Ambler class A extended-spectrum $\beta$ lactamases in Pseudomonas aeruginosa: novel developments and clinical impact. Antimicrob Agents Chemother 47, 2385-2392. 